\title{
Vergleich von Sprichwörtern und Redewendungen über Tiere im Deutschen und Arabischen.
}

\author{
Dr. Ashraf Mohamed Samir
}

Al-Alsun Fakultät

Beni-Suef Universität 



\section{Einführung}

In der deutschen und arabischen Sprache gibt es viele Sprichwörter und Redewendungen über Tiere, die in der Gesamtbedeutung und der Einzelbedeutung der Wörter sehr ähnlich sind. Es gibt Redewendungen, bei denen sich die Bedeutung ein wenig ändert, dennoch sind die Charaktere der Tiere sich ähnlich. Oder es gibt Sprichwörter, in denen die Bedeutung in völlig anderen Worten ausgedrückt wird, die nur im Deutschen oder im Arabischen gelten. Redewendungen und Sprichwörter über Tiere spiegeln die langjährige menschliche Beobachtung der Gewohnheiten von Tieren und deren Aussehen wieder, vermitteln aber auch unsere Einstellung gegenüber Tieren.

So reflektieren Sprichwörter und Redewendungen mit Tiernamen im Deutschen und Arabischen: - körperliche Fähigkeiten und Qualitäten: schwach wie ein Huhn, stark oder schnell wie ein Pferd, schwimmen wie ein Fisch, flink wie ein Affe, scharfsichtig wie ein Luchs oder ein Adler, der Geruchsinn eines Hundes und viele andere mehr.

Aussehen: Ziegenbart, schwarz wie ein Rabe, mit einer Sperlingsnase, dick wie ein Schwein usw.; Eigenschaften der 
Psyche (oder Charaktereigenschaften): stur wie ein Esel, ein Stier, ausgeruht wie ein Widder, dumm wie eine Gans und listig wie die Schlangen, übermütig, hochnäsig, eitel, stolz, hochmütig wie ein Hahn usw.; - intellektuelle Qualitäten: schlau wie ein Fuchs, treu wie ein Hund, klug wie ein Wolf usw.; Fähigkeiten, Gewohnheiten: stumm wie ein Fisch, wiederholen wie ein Papagei, Vogel-Strauß-Politik usw.

Merkmale, die eine Person Tieren zuschreibt, können in verschiedenen Sprachen gleichbedeutend sein oder sich erheblich unterscheiden. So wird beispielsweise das Bild eines Vogels in einem arabischen Sprichwort durch das Bild einer Fliege in einem deutschen Sprichwort ersetzt, z.B.: Zwei Fliegen mit einer Klappe schlagen يضرب عصفورين بحجر und das Bild eines Fisches durch das Bild einer Katze, z.B.: die Katze nicht im Sack kaufen يشترى سمك في مياه .

Und doch nehmen die Deutschen das Verhalten von Tieren vielleicht anders wahr als wir Ägypter? Gibt es spezielle Traditionen, nach denen sich Tiere in ihren Stimmen unterscheiden, oder haben sie (Tiere) unterschiedliche Gewohnheiten in der arabischen Kultur und in der deutschen? Um Antworten auf diese Fragen zu finden, wenden wir uns Redewendungen und Sprichwörtern zu. Wir gehen davon aus, 
dass Deutsche und Ägypter im Allgemeinen in etwa die gleiche Einstellung zu Tieren haben sollten. Dennoch soll hier versucht werden, etwaige Unterschiede zu finden.

ملخص العربى يوجد في اللغتين الألمانية والعربية العديد من الأمثال والتعابير عن الحيوانات المتشابهة جدًا في المعنى العام والمعنى الفردي للكلمات. هناك تعابير يتغير فيها المعنى قليلاً ،

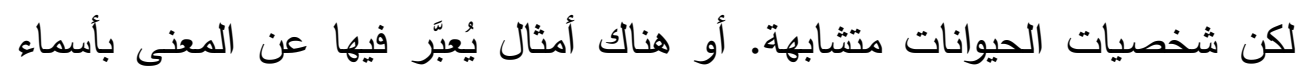
حيوانات مختلفة تمامًا تتطبق فقط باللغة الألمانية أو العربية .تعكس التعابير والأمثال عن الحيوانات الملاحظة البشرية طويلة الأمد لعادات الحيوانات ومظهرها ، ولكنها تنقل أيضًا موقفنا تجاه الحيوانات. الأمثال والأقوال المأثورة التي تحمل أسماء حيوانات باللغتين الألمانية والعربية تعكس: - القدرات والصفات الجسدية: ضعيف كالاجاج ، قوي أو سريع كالحصان ، يسبح كالسمك ، رشيق كالقرد ، شديد النظر كالنسر وغيرها الكثير.

- المظهر : أسود مثل غراب ، لديه أنف عصفور ، سمين مثل الخنزير ، إلخ ؛

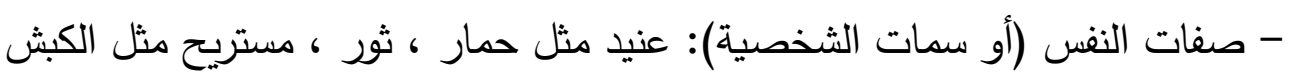
، غبي مثل أوزة ومكار مثل الثعابين ، ، متعجرف مثل الديك ، إلخ ؛ - الصفات الفكرية: ماهر كالثعلب ، مخلص كالكلب ، ذكي كالذئب ، إلخ ؛ -المهارات والعادات: صامت مثل السمكة ، والتكرار مثل البيغاء ، وسياسة النعام ،

قد تكون الخصائص التي ينسبها الشخص إلى الحيوانات مترادفة في لغات مختلفة أو قد تختلف اختلافًا كبيرًا. على سبيل المثال ، تم استبدال صورة طائر في مثل عربي بصورة ذبابة في مثل ألماني ، على سبيل المثال: يضرب ذبابتين بمنشة فى اللغة 
الالمانية ،أما فى اللغة العربية : يضرب عصفورين بحجر وصورة سمكة بصورة قطة ، على سبيل المثال. : فى اللغة الالمانية : يشترى القطة فى شوال (كيس) وفى اللغة العربية : يشترى سمك في مياه.

ومع ذلك ، ربما ينظر الألمان إلى سلوك الحيوان بشكل مختلف عن تصورنا نحن المصريين؟ أم أن للحيوانات عادات مختلفة في الثقافة العربية وفي الثقافة الألمانية؟

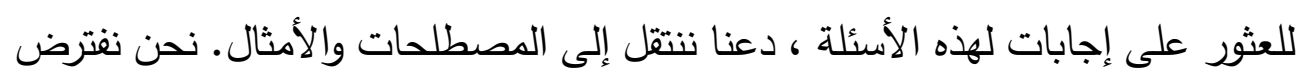
أن الألمان والمصريين بشكل عام يجب أن يكون لديهم نفس الموقف تقريبًا تجاه الحيوانات. ومع ذلك ، يجب بذل محاولة هنا للعثور على أي اختلافات.

\section{Einleitung}

Ein Sprichwort im Mund wiegt hundert Pfund. - Im Sprichwort wird die Wahrheit gesagt. Zurzeit werden zusammen mit der Entwicklung der Technologie Informationen auf verschiedene Weise gespeichert und übertragen. Die einfachste und zugänglichste Art, Erfahrungen zu vermitteln, ist jedoch die gesprochene Sprache.

In der deutschen und arabischen Sprache gibt es viele Sprichwörter und Redewendungen über Tiere, die in der Gesamtbedeutung und der Einzelbedeutung der Wörter sehr ähnlich sind. Es gibt Redewendungen, bei denen sich die Bedeutung ein wenig ändert, dennoch sind die Charaktere der Tiere sich ähnlich. Oder es gibt Sprichwörter, in denen die 
Bedeutung in völlig anderen Worten ausgedrückt wird, die nur im Deutschen oder im Arabischen gelten. Redewendungen und Sprichwörter über Tiere spiegeln die langjährige menschliche Beobachtung der Gewohnheiten von Tieren und deren Aussehen wieder, vermitteln aber auch unsere Einstellung gegenüber Tieren.

So reflektieren Sprichwörter und Redewendungen mit Tiernamen im Deutschen und Arabischen: - körperliche Fähigkeiten und Qualitäten: schwach wie ein Huhn, stark oder schnell wie ein Pferd, schwimmen wie ein Fisch, flink wie ein Affe, scharfsichtig wie ein Luchs oder ein Adler, der Geruchsinn eines Hundes und viele andere mehr.

Aussehen: Ziegenbart, schwarz wie ein Rabe, mit einer Sperlingsnase, dick wie ein Schwein usw.; Eigenschaften der Psyche (oder Charaktereigenschaften): stur wie ein Esel, ein Stier, ausgeruht wie ein Widder, dumm wie eine Gans und listig wie die Schlangen, übermütig, hochnäsig, eitel, stolz, hochmütig wie ein Hahn usw.; - intellektuelle Qualitäten: schlau wie ein Fuchs, treu wie ein Hund, klug wie ein Wolf usw.; Fähigkeiten, Gewohnheiten: stumm wie ein Fisch, wiederholen wie ein Papagei, Vogel-Strauß-Politik usw. 
Merkmale, die eine Person Tieren zuschreibt, können in verschiedenen Sprachen gleichbedeutend sein oder sich erheblich unterscheiden. So wird beispielsweise das Bild eines Vogels in einem arabischen Sprichwort durch das Bild einer Fliege in einem deutschen Sprichwort ersetzt, z.B.: Zwei Fliegen mit einer Klappe schlagen يضرب عصفورين بحجر und das Bild eines Fisches durch das Bild einer Katze, z.B.: die Katze nicht im Sack kaufen يشترى سمك في مياه ·

Und doch nehmen die Deutschen das Verhalten von Tieren vielleicht anders wahr als wir Ägypter? Gibt es spezielle Traditionen, nach denen sich Tiere in ihren Stimmen unterscheiden, oder haben sie (Tiere) unterschiedliche Gewohnheiten in der arabischen Kultur und in der deutschen? Um Antworten auf diese Fragen zu finden, wenden wir uns Redewendungen und Sprichwörtern zu. Wir gehen davon aus, dass Deutsche und Ägypter im Allgemeinen in etwa die gleiche Einstellung zu Tieren haben sollten. Dennoch soll hier versucht werden, etwaige Unterschiede zu finden.

\section{Ziel der Forschung:}

Der Zweck dieser Studie: Redewendungen und Sprichwörter über Tiere in der arabischen und deutschen Sprache zu sammeln. 
Einen Vergleich zwischen den Sprichwörtern in beiden Sprachen zu machen, dabei können wir die Identität und Charakteristik der deutschen oder arabischen Sprache hervorheben. Das Ziel der vorliegenden Arbeit besteht hauptsächlich darin, die Besonderheiten der Sprichwörter im Deutschen und Arabischen zu erforschen.

\section{Forschungshypothese:}

In der deutschen und arabischen Sprache gibt es eine große Anzahl von Sprichwörtern über Tiere, die in ihrer Bedeutung und lexikalischen Bedeutung der Wörter sehr ähnlich sind. Es gibt sicherlich Sprüche, bei denen die Bedeutung ein wenig variiert, aber die Charaktere der Tiere sind ähnlich. Oder es gibt Sprichwörter, in denen die Bedeutung in völlig anderen Worten ausgedrückt wird, die nur im Deutschen oder Arabischen gelten.

\section{Definition des Sprichwortes}

Der Begriff Sprichwort besteht aus zwei Teilen, Sprich und Wort. Die eigenen durchgeführten Recherchen zeigten, dass Sprichwort das einzige Kompositum im Deutschen mit dem Wort Sprich ist, sonst wird nur der Wortstamm Sprech in anderen Wortzusammensetzungen gefunden, wie Sprechchor, Sprechakt, Sprechanlage, Sprechgesang, ... 
J. Grimm dachte an einen engen Zusammenhang mit sprechen als Verb und glaubte Sprichwort wäre entstellt aus Sprechwort. ${ }^{1}$

Wir fassen das Wort jetzt in einem engeren Sinne und verstehen unter Sprichwörtern: im Volksmund umlaufende, in sich geschlossene Sprüche von lehrhafter Tendenz und gebobener Form. Durch diese Begriffsbestimmung ist die Grenze gezogen einerseits gegen die sprichwörtlichen Redensarten. Diese sind zwar volkläufig, aber weder lehrhaften Charakters noch in sich geschlossen, und von den formgebenden Kunstmitteln steht innen nur die Bildlichkeit zur Verfügung, z. B. im Trüben fischen, Öl ins Feuer gießen, an den Bettelstab kommen. Andererseits gegen die Sinnsprüche und Sentenzen. Diese sind zwar in sich geschlossen und lehrhaft, haben auch in der Regel eine gehobene Form, sind aber nicht volkläufig. ${ }^{2}$

\section{Zum Begriff „, Sprichwort“}

Die deutsche Sprache ist reich an Sprichwörtern und Sprüchen. Jede Nation hat ihre eigenen Sprichwörter und Sprüche, die einen wichtigen Teil des nationalen Kulturerbes bilden. Viele Sprichwörter in arabischer und deutscher Sprache haben das gleiche Wesen, das jedoch aufgrund der Tatsache, dass das

${ }^{2}$ Vgl. Seiler:1967,S. 2. 
Sprichwort auf unterschiedlichen Assoziationen basiert, in völlig unterschiedlichen Worten vermittelt werden kann. Allerdings haben nicht alle vorhandenen Sprichwörter genaue oder enge Übereinstimmungen in anderen Sprachen, denn die Geschichte der Entwicklung jeder Nation ist individuell.

Sprichwörter und Sprüche spiegeln die jahrhundertealte Volksweisheit wider und sind in der Regel leicht zu merken.

Der größte Reichtum eines Volkes ist seine Sprache! Jahrtausende sammeln sich an und leben für immer in dem Wort unzählige Schätze menschlichen Denkens und Erlebens. Sprichwörter sind seit der Antike zu uns gekommen. Dies sind kurze, einfache, aber nachdenkliche Sprüche. Sie wurden vom Volk gebildet, noch bevor der Brief in Arabisch erschien. Ein Volk, das weder lesen noch schreiben kann, als würde es eine eigene mündliche Schule gründen. In ihren besten Sprichwörtern lehrten die Menschen von Vätern zu Söhnen, von Großvätern zu Enkelkindern ihre geschätzten Lebensregeln und lehrten die Kinder den Geist. Es war immer möglich, nützliche Ratschläge in innen zu finden, was in diesem oder jenem Fall des Lebens zu tun ist, weil sie alle Aspekte des Lebens der Menschen abdecken und populäre Vorstellungen darüber widerspiegeln Natur, über 
Heimat, über Arbeit, über Familie, über Moral, über Verstand und Dummheit, über Gut und Böse usw.

Alte Sprichwörter leben heute in unserer Sprache: sowohl im Gespräch als auch in Büchern. Sprichwörter schmücken unsere Rede, machen sie lebendig, witzig.

Verwenden wir in unserer Umgangssprache viele Sprichwörter, Sprüche, Witze und humorvolle Sprüche? Wie viele Sprichwörter - diese Klumpen der Volksweisheit - kennt jeder von uns auswendig? Wir glauben, dass wir uns nicht irren werden, wenn wir als größte die Zahl 50 angeben (und in der gewöhnlichen Umgangssprache verwenden wir vielleicht sogar weniger). Und dies ist, während der unerschöpfliche Reichtum unserer Sprichwörter und Sprüche Tausende von ihnen umfasst.

Sprichwörter entwickeln die Fähigkeit, ein Urteil in einem klingenden Wort auszudrücken, und seine Einstellung zu dem Lebensphänomen, das dieses Urteil verursacht hat. Es ist ein Urteil über das Leben, das Ergebnis von Beobachtungen des Lebens, eine Schlussfolgerung aus einigen Tatsachen. Das Sprichwort spiegelt die tiefen und komplexen Zusammenhänge verschiedener Lebensphänomene wider und soll den Hörer in etwas überzeugen, etwas fordern, billigen, loben oder umgekehrt 
lächerlich machen. Ein Sprichwort drückt einen vollständigen Gedanken aus, enthält Anweisungen und besteht aus einem, zwei und manchmal mehr Sätzen.

In Sprichwörtern ist es einfach, das Konzept des Subtextes zu formulieren, ohne den Begriff selbst zu benennen. Darin ist jedes Wort wichtig, und fast jedes Wort wird logisch betont. Ein typisches Zwei-Term-Sprichwort hat normalerweise zwei Hauptspannungen in jedem Teil . In einem Zwei-TermSprichwort ist der zweite Teil in den meisten Fällen besonders wichtig, da er die Idee ausdrückt, die das Wesen des Sprichworts ausmacht, und im ersten Teil wird das Thema genannt beurteilt oder gibt den Zustand der Handlung an, die Ursache des Phänomens.

Volkskunst und unerschöpfliche mündliche Volkskunst, die den modernen Menschen in die Welt des künstlerischen Denkens einführt, das öffentliche Bewusstsein von Menschen aus den entferntesten Epochen und Zeiten, die uns nahestehen. Märchen, Rituallieder, Verschwörungen und Wahrsagerei haben ihre Wurzeln in den tausendjährigen Tiefen der Geschichte. Zusammen mit den Menschen und ihrer Sprache wird ein Sprichwort geboren, die Menschen leben danach; Darin liegen seine Erfahrungen, weltliche Erfahrungen, seine Philosophie. 
Seit vielen Jahrhunderten haben sich in der Sprache des Volkes aphoristische Sprüche und bildliche Ausdrücke angesammelt, die wir als Sprichwörter und Sprüche kennen.

Der Übergang von Generation zu Generation, Sprichwörter und Sprüche unterstützten die Lebensweise der Menschen, stärkten das geistige und moralische Image der Menschen und regulierten das Leben eines jeden gewöhnlichen Menschen.

Sprichwörter und Sprüche sind das älteste und beliebteste Genre der mündlichen Volkskunst, die Folklore. Folklore - aus dem Englischen. Folklore - Volksweisheit, Volkswissen) - die künstlerische kollektive schöpferische Tätigkeit des Volkes, die sein Leben, seine Ansichten, Ideale widerspiegelt; vom Volk geschaffen und unter den Massen existierend. So spiegelten die Menschen in Sprichwörtern und Sprüchen ihre Einstellung zur Natur und ihren Phänomenen wider, die soziale und historische Erfahrung ihrer Vorfahren, drückten ihre Weltanschauung, moralischen Standards und ästhetischen Ideale aus.

\section{Zum Begriff „Redensart“}

Öfter noch als Sprichwörter werden in der Umgangs- und auch in der Literatursprache stehende Wendungen gebraucht, die der Rede eine gewisse Frische, Natürlichkeit oder Derbheit geben. 
Man nennt sie, weil sie mit den Sprichwörtern verwandt und wie diese volkläufig sind, sprichwörtliche Redensarten. Zu ihnen sind einmal die kurzen Ausrufe zu rechnen, durch die man im alläglichen Leben einer Stimmung kräftigen Ausdruck zu geben liebt. [ . . ] Weit wichtiger indessen ist die große Masse derjenigen sprich-wörtlichen Redensarten, die bei ihrem Gebrauch der Einsetzung eines Satzgliedes bedürfen, ohne welches sie unvollständig und gar sinnlos sind. [. . .] Die sprichwört-lichen Redensarten sind nicht durch Kürzung voller Sprichwörter entstanden, und ebensowenig die Sprichwörter durch Erweiterung sprichwörtlicher Redensarten. Beide stehen vielmehr von Anfang an nebeneinander, wie auch ihre psychologische Ursache dieselbe ist, nämlich der Wunsch des Sprechenden, schlagkräftig, eindrucksvoll und wenn möglich bildlich-anschaulich zu reden. ${ }^{3}$

Redensarten sind kleinere Bausteine, ja sie sind in gewisser Hinsicht der Mörtel, der überall eingefügt werden kann. Das Sprichwort ist ein größerer Baustein; es ist schwerer einzufügen. Es ist seltener, aber es hat auch wesentlich mehr Gewicht. ${ }^{4}$ Sprichwörtliche Redensarten sind also bildliche Wendungen, in denen nicht ein einzelner Begriff, sondern eine ganze

\footnotetext{
${ }^{3}$ Vgl.Friedrich Seiler, S. 11

${ }^{4}$ Vgl.Hermann Bausinger, S 94-95
} 
Gedankenfolge durch eine sinnlich-bildliche Wendung ausgedrückt (nicht beschrieben) wird. Sie sind kaum durch weniger bildliche Wendungen zu ersetzen; ihre einzelnen Wörter sind nicht austauschbar. Sie treten in einer festen Prägung auf, die beim Gebrauch sprachlich nicht verändert wird; sie bilden selbst aber keinen Satz, sondern werden erst durch die Einbeziehung in einen Satz aktiv benutzbar. Sie sind volksläufig, sie werden also von allen Schichten des Volkes in etwa gleicher Weise benutzt. Ihre Bedeutung als sprachliche Einheit hat sich meist so weit von der ursprünglichen Bedeutung der Einzelwörter entfernt, dass ihr eigentlicher Sinn gar nicht oder kaum mehr empfunden wird.[ · ]

Die sprichwörtlichen Redensarten haben, im Gegensatz zu den Sprichwörtern, keine lehrhafte Tendenz; sie dienen als formales Mittel der Rede und geben ihr Frische und Ausdruckskraft. ${ }^{5}$

Das »Sprichwörtliche» einer Redensart liegt darin, dass sie in ihrem Wortlaut relativ konstant ist. [. . . ] Redensarten zeigen eine relativ starre Formulierung, die nur selten den Ersatz bedeutungsähnlicher Wörter oder Erweiterungen durch andere Wörter gestattet. Man spricht deshalb auch von »stehenden« 
Redensarten. Die Redensart ist also ein Bildwort in überlieferter Ausdrucksform. ${ }^{6}$

\section{Sprichwörter über Tiere auf Deutsch und Arabisch.}

\subsection{Sprichwörter auf Deutsch und Arabisch, die dieselbe} Bedeutung und denselben Wortschatz haben

Deutsche und arabische Sprichwörter haben die gleiche Bedeutung und beziehen sich auf die gleichen Tiere. Es gibt so viele Sprichwörter, die sowohl auf Deutsch, als auch auf Arabisch verwendet werden z.B.:

\begin{tabular}{|c|c|}
\hline Deutsch & Arabisch \\
\hline $\begin{array}{l}\text { Wenn die Katze aus dem Haus ist, } \\
\text { tanzen die Mäuse auf dem Tisch. }\end{array}$ & إن غاب القط ، العب يا فار \\
\hline Eine Katze hat neun Leben & زي القطط بسبع أرواح \\
\hline Krokodiltränen weinen & 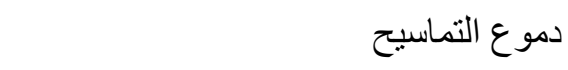 \\
\hline 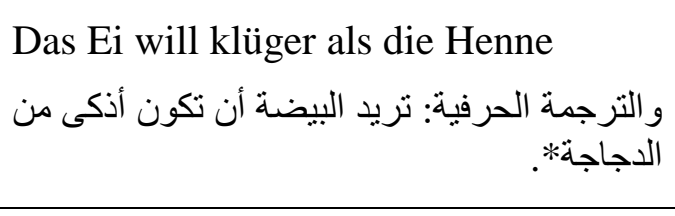 & 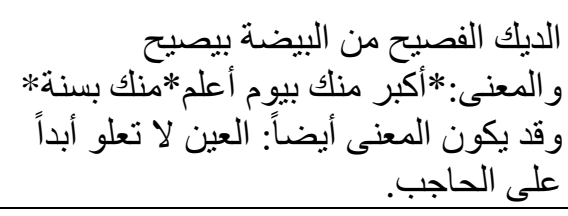 \\
\hline der Hahn im Korb sein & فرخة بكثك / ديك البر ابر \\
\hline Ein Hühnchen zu rupfen haben & 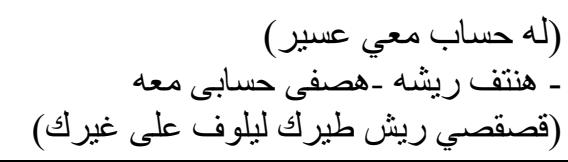 \\
\hline Hunde, die bellen, beißen nicht. & الكلاب التي تعوي لاتعض \\
\hline
\end{tabular}

${ }^{6}$ Vgl.Lutz Röhrich, S.9 


\begin{tabular}{|c|c|}
\hline $\begin{array}{l}\text { Wenn du einen Freund brauchst, kaufe } \\
\text { dir einen Hund. }\end{array}$ & الكلب هو أوفى صديق للإنسان \\
\hline $\begin{array}{l}\text { Die Hunde bellen, die Karawane zieht } \\
\text { weiter. }\end{array}$ & الكلاب تنبح، القافلة تسير. \\
\hline $\begin{array}{l}\text { Den Kranken ärgert die Fliege an der } \\
\text { Wand. }\end{array}$ & (تز عجه كل صغيرة وكبيرة) \\
\hline Der hat doch einen Vogel & مخه طار \\
\hline $\begin{array}{l}\text { Gib einem Armen keine Fische, aber } \\
\text { lehre ihn das Fischen. }\end{array}$ & 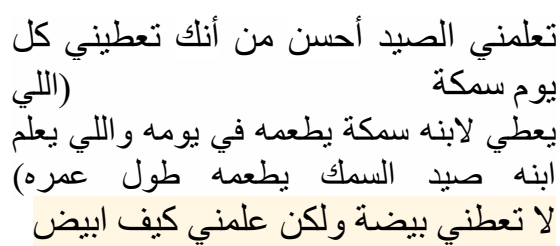 \\
\hline
\end{tabular}

4.2. Deutsche und arabische Sprichwörter, die gleiche Bedeutung haben, aber mit unterschiedlichen Tieren

\begin{tabular}{|c|c|}
\hline Deutsch & Arabisch \\
\hline Zwei Fliegen mit einer Klappe schlagen & يضرب عصفورين بحجر \\
\hline Des Pudels Kern & عارف » القرد أو العفريت مخبي ابنه فين \\
\hline Die Katze im Sack kaufen & يشترى سمك في مياه \\
\hline Eine dumme Gans & غبي كالحمار - "ثور لاهٍ في برسيمه" \\
\hline wie ein Elefant im Porzellanladen & مثل الثور الهائج "ثور لاهٍ في برسيمه" \\
\hline den Vogel abschießen & جاب الديب من ديله \\
\hline jemandem einen Bären aufbinden & كذاب كذب الإبل \\
\hline Da liegt der Hund begraben. & هنا مربط الفرس \\
\hline
\end{tabular}




\begin{tabular}{|c|c|}
\hline $\begin{array}{l}\text { aus einer Mücke einen Elefanten } \\
\text { machen }\end{array}$ & 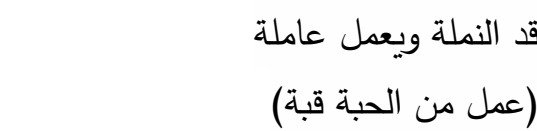 \\
\hline Das kann kein Schwein lesen & نكش فراخ (لا يمكن قراءته) \\
\hline Eine Schwalbe macht noch keinen Sommer & لابصياح الغراب يأتي المطر \\
\hline Wie von der Tarantel gestochen & قرصته عقربة \\
\hline $\begin{array}{l}\text { Jeder Hahn ist König auf seinem Mist } \\
\text { " كل ديك على مزبلته صيباح " }\end{array}$ & الكلب فى بيته سبع \\
\hline $\begin{array}{l}\text { Die Katze lässt das Mausen nicht. } \\
\text { (Immer wieder wird die Katze auf } \\
\text { Mäusejagd gehen, man kann es ihr nicht } \\
\text { abgewöhnen.) }\end{array}$ & 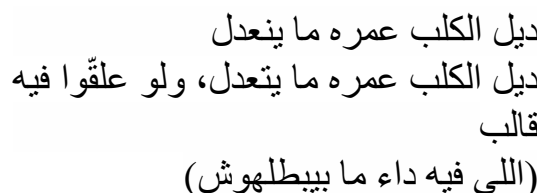 \\
\hline
\end{tabular}

\subsection{Deutsche tierische Sprichwörter und ihre entsprechenden arabischen Sprichwörter, die im Arabischen keinen Tieren entsprechen.}

\begin{tabular}{|c|c|}
\hline Deutsch & Arabisch \\
\hline Jemandem einen Floh ins Ohr setzen & الزن على الأذن \\
\hline 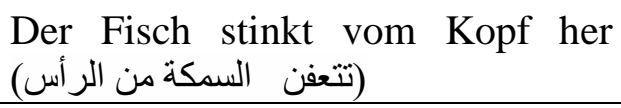 & حراميهال عن الفساد السياسي او اقتصادي) حاميها \\
\hline Kleinvieh macht auch Mist & "القطيع الصغير يخلف فضلات كثيرة" \\
\hline $\begin{array}{l}\text { Mit Speck fängt man Mäuse } \\
\text { بالطعم الجيد نصطاد الفئران }\end{array}$ & أطعم الفم تستحى العين \\
\hline
\end{tabular}




\begin{tabular}{|c|c|}
\hline 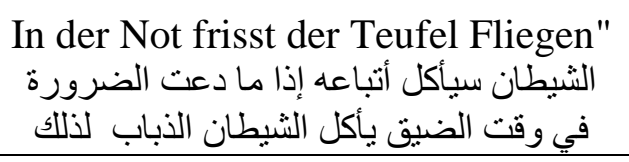 & الجوع كافر \\
\hline $\begin{array}{l}\text { Einem geschenkten Gaul schaut } \\
\text { man nicht ins Maul. } \\
\text { "لا ينظر المرء في فم حصان مُهدى" }\end{array}$ & 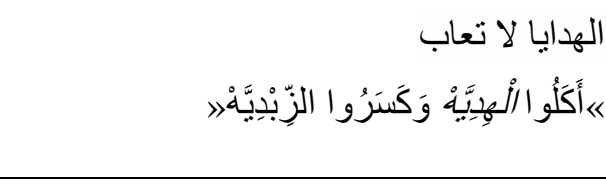 \\
\hline $\begin{array}{l}\text { Die Ratten verlassen das sinkende } \\
\text { Schiff } \\
\text { تهرب الجرذان من السفينة الغارقة }\end{array}$ & "أنا أولاً ومن الجبن و الأنانية \\
\hline $\begin{array}{l}\text { "Was Hänschen nicht lernt, lernt } \\
\text { Hans nimmermehr" } \\
\text { ما لم تتعلمه الصيصان، لن تتقنه الدجاجات }\end{array}$ & 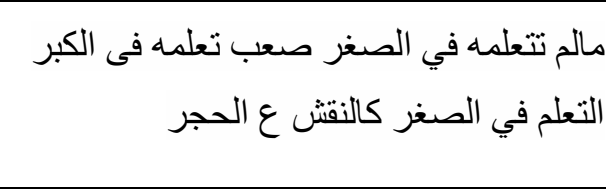 \\
\hline $\begin{array}{l}\text { aus einer Mücke einen Elefanten } \\
\text { machen }\end{array}$ & عمل من الحبة قبة \\
\hline sich zum Affen machen & يجعل من نفسه أضحوكة أمام الناس \\
\hline $\begin{array}{l}\text { Da liegt der Hund begraben } \\
\text { (dies ist die Ursache der } \\
\text { Schwierigkeiten) }\end{array}$ & هذا هو سبب المشاكل ,المتاعب \\
\hline $\begin{array}{l}\text { Der Wolf im Schafspelz } \\
\text { ذئب في ملابس شاة }\end{array}$ & 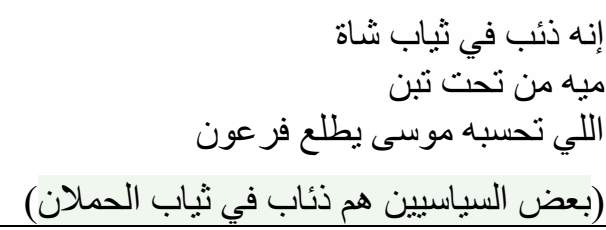 \\
\hline Ein alter Hase sein & رجل ذو حكمة \\
\hline $\begin{array}{l}\text { Auch ein blindes Huhn findet mal } \\
\text { ein Korn. } \\
\text { حتى الدجاج الاعمي يجد أحيانا حبة }\end{array}$ & 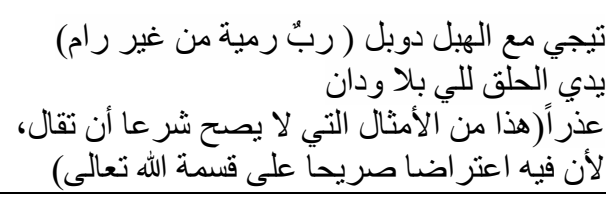 \\
\hline $\begin{array}{l}\text { Der frühe Vogel fängt den Wurm. } \\
\text { الطائر المبكر يمسك بالدودة }\end{array}$ & 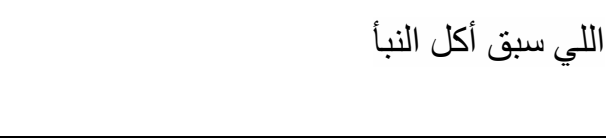 \\
\hline Einen Kater haben & عنده صداع \\
\hline
\end{tabular}




\begin{tabular}{|c|c|}
\hline Die Kuh vom Eis holen & يخر العجين) شخص من مشكلته ( يطلعه زى الثعرة \\
\hline $\begin{array}{l}\text { Die Schweine von heute sind die } \\
\text { Schinken von morgen } \\
\text { خنازير اليوم هي لحم طعام الغد }\end{array}$ & 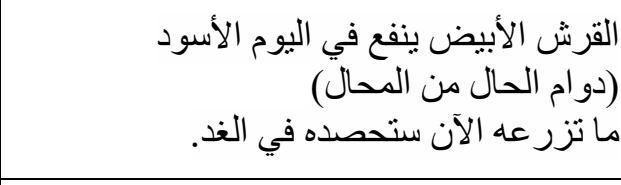 \\
\hline $\begin{array}{l}\text { Elternliebe ist oft Affenliebe } \\
\text { حب الو الدين يثبه غالبا حب القردة لبعضها البعض } \\
\text { Affenliebe als jede Form der } \\
\text { übertriebenen Liebe }\end{array}$ & حب الو الدين لا يضاهيه حب \\
\hline $\begin{array}{l}\text { Beschert Gott den Hasen, beschert } \\
\text { er auch den Rasen. } \\
\text { إذا أعطى الله الأرنب ، فإنه يعطي العشب أيضا }\end{array}$ & 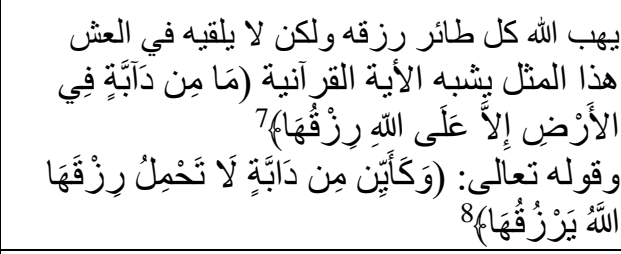 \\
\hline $\begin{array}{l}\text { Sei kein } \quad \text { Frosch! } \\
\text { Stell dich der Situation und tauch } \\
\text { nicht einfach ab. Du kannst das! }\end{array}$ & (ماتبقاش جبان) \\
\hline "Klappe zu, Affe tot" & خلص الكلام \\
\hline $\begin{array}{l}\text { Das Ei will klüger sein als die } \\
\text { Henne. }\end{array}$ & العين لا تعلو أبداً على الحاجب \\
\hline $\begin{array}{l}\text { Eine blinde Henne findet } \\
\text { aucheinmal ein Korn. }\end{array}$ & 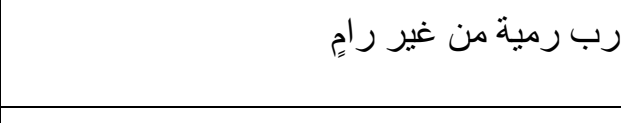 \\
\hline Eulen nach Athen tragen & باع الماء في حارة السقّائين ــا \\
\hline Mein Name ist Hase & ثور لاهٍ في برسيمه ( اللي مايعرفش يقول عدس) \\
\hline 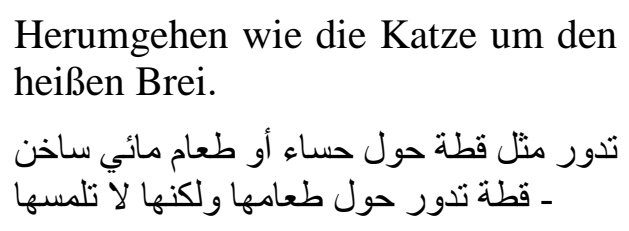 & 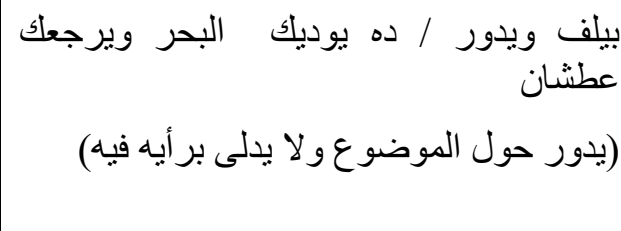 \\
\hline
\end{tabular}

${ }^{7}$ Vgl. Der edle Quran, Sura Hud, Vers 6, Seite 222

${ }^{8}$ Vgl. Der edle Quran, Sura die Spinne, Vers 60, Seite 403 


\begin{tabular}{|c|c|}
\hline Das ist ein Katzensprung & فركة كعب \\
\hline $\begin{array}{l}\text { Wo sich Fuchs und Hase gute Nacht } \\
\text { sagen } \\
\text { الدكان الذي يقول فيه الذئب والارنب طابت ليلتك }\end{array}$ & خطرة بي حالة الذهاب الي اماكن بعيده خالية \\
\hline $\begin{array}{l}\text { Wo sich Fuchs und Hase gute Nacht } \\
\text { sagen } \\
\text { (weit von den Menschen, außerhalb } \\
\text { einer Stadt) }\end{array}$ & ودوه وراء الثمس \\
\hline $\begin{array}{l}\text { Ein Fisch auf dem Trockenem } \\
\text { سكة على اليابسة }\end{array}$ & المناسب له لو مكف حانك الصح شخص لبس في المكان \\
\hline
\end{tabular}

\subsection{Arabische tierische Sprichwörter und ihre}

\section{entsprechenden deutschen Sprichwörter}

\begin{tabular}{|c|c|}
\hline Arabisch & Deutsch \\
\hline ما كل من ركب الحصان خيال & $\begin{array}{l}\text { Es sind nicht alles Köche, die lange } \\
\text { Messer tragen }\end{array}$ \\
\hline حمار تلك العرجاء و لا سؤ اللك اللئيم & Klein, aber mein \\
\hline سكتنا له فذخل بحماره & $\begin{array}{l}\text { Wenn man dem Teufel den kleinen } \\
\text { Finger gibt, dann nimmt der Teufel } \\
\text { die ganze Hand. }\end{array}$ \\
\hline قال هيسخطوك يا قرد ،قال ما هي كده كده & Er hat nichts zu verlieren \\
\hline الطيورُ على أثنكالها تقع & Gleich und gleich gesellt sich gern. \\
\hline ابن الوز عوام & $\begin{array}{l}\text { Der Apfel fällt nicht weit vom } \\
\text { Stamm }\end{array}$ \\
\hline
\end{tabular}

Vielen Tieren werden bestimmte menschliche Eigenschaften nachgesagt. Oder anders herum: für diverse menschliche 
Eigenschaften stehen die in der untenstehenden Tabelle genannten Tiere als Symbol. ${ }^{9}$

Einiges davon trifft mit Sicherheit zu. So ist die Vermehrungsrate bei Kaninchen wissenschaftlich nachgewiesen sehr hoch. Das Kaninchen als Symbol der Fruchtbarkeit ist daher nachvollziehbar. Auch die Wildheit und Gefährlichkeit eines Tigers steht außer Frage. Einen Wurm mit der negativen Eigenschaft der Kriecherei $\mathrm{zu}$ assoziieren ist durchaus nachvollziehbar. Eine Biene ist in ihrem kurzen Leben immer unterwegs und sammelt emsig Nektar und Pollen. Da liegt es nahe, dieses Insekt mit dem Prädikat „sehr fleissig“ zu versehen.

\section{Tier-Symbolik}

Die Tier-Symbolik erklärt die verschiedenen Eigenschaten der Menschen ${ }^{10}$

Der Löwe symbolisiert Mut, Führung und Würde. Er es ist der König der Wälder und des Kampfs, die er mit diesen Eigenschaften symbolisiert. Auch gesagt wird z.B. mutiger als ein Löwe, stärker als ein Löwe oder kühner als ein Löwe. Ein Hund, der rennt, ist besser als der hockende Löwe und sucht nach

\footnotetext{
${ }^{9}$ Vgl. http://www.kuriosetierwelt.de/tier-symbolik-bienen-sind-fleissig-und-esel-sinddumm/

${ }^{10} \mathrm{Vgl}$. http://www.kuriosetierwelt.de/tier-symbolik-bienen-sind-fleissig-und-esel-sinddumm/
} 
einem Fasan, der im Käfig eines Löwen steht sind Andere im Arabischen Kulturraum gebrauchte Redewendungen. In Deutschland steht der Löwe für Macht und Kraft.

\section{-Elefanten}

Der Elefant ist ein Symbol für Stärke enorme Größe, Gier und ein Mittel des Krieges, und er trifft diejenigen, die er symbolisiert, mit diesen starken und riesigen Eigenschaften (wie ein Elefant), und es wird auch der Ausspruch, wie ein tobender Elefant oder etwa ein Elefantenfresser und ein Feuerfresser schlagen Kannibalen. Auch wurde er im Heiligen Koran als erwähnt in der Sure der Elefant. Im Deutschen, gibt es keine Redewendungen, den Elefanten betreffend.

\section{- Kamel}

Das Kamel, bedeutet Leichtigkeit der Unterwerfung, Hass und Geduld (von Hunger, Durst und Schaden), was für jeden, der diese Eigenschaften hat, positiv ist. Es gibt Redewendungen, wie nur der Lohn des Jungen, nicht des Kamels. Es wird auch gesagt, sie haben ein Kamel gestohlen. Im Koran wird das Kamel auch mehrmals erwähnt. Im Deutschen gibt es „Eher geht ein Kamel durch ein Nadelöhr als dass ein Reicher ins Reich Gottes kommt", das Zitat stammt aus der Bibel. 
-Das Pferd

Das Pferd und ist ein Zeichen von Originalität, Großzügigkeit und Schönheit und des Weiteren ein Mittel der Kriegsführung und Frömmigkeit. Es wurde gesagt, das Pferd ist für diejenigen, die es reiten, und das Schwert ist für den, der es trägt wie die Posaune. Auch das Pferd wird im Heiligen Koran erwähnt.

- Das Maultier

Das Maultier steht für Sturheit, Stärke, Steifheit, blinden Gehorsam, mangelnde Originalität und das Bocken. Im Arabischen wird auch gesagt, der Körper eines Maultiers und die Träume eines Vogels. Im Koran wird der Name des Maultiers erwähnt. In Deutschland wird gerne der Ausdruck „störrisch wie ein Maultier verwendet"

Der Affe:

Im Arabischen wird gesagt wird auch gesagt, der Affe ist hässlich, aber schön oder der Affe ist ein Reh im Auge seiner Mutter. Der Affe wird im Heiligen Koran erwähnt. In Deutschland steht der Affe für Dummheit und Eitelkeit.

\section{- Der Wolf}

Der Wolf gilt im arabischen Kulturraum als verräterisch und unersättlich (er steht auch für, Tyrannei, Einsamkeit, Hunger und 
Mut, der keine Gnade kennt). In Deutschland, gilt er als Böse, aber auch als guter Kämpfer

- Die Schweine

Das Schwein, für sexuelle Übergriffe, Lust und Unreinheit. In Deutschland, steht das Schwein, zwar auch für Unreinheit im Sinne Unordnung unsauber und charakterlich verdorben, aber auch für Glück.

\section{-Der Esel}

Der Esel steht für Dummheit, Mangel an Gedanken, Geduld und eine unangenehme Stimme. Aber wegen der Fähigkeit des Esels, sein Zuhause auch nachts zu finden, und der Fähigkeit, zwischen dem Geräusch, das inn stoppt, und dem Geräusch, das ihn zum Gehen auffordert, zu unterscheiden, wird manchen Menschen nachgesagt, dümmer als ein Esel zu sein, da sie, dazu nicht in der Lage seien. In Deutschland, steht der Esel auch für Dummheit.

\section{- Der Hund}

Der Hund steht für Loyalität, Gehorsam und Kühnheit und er wird auch "der treue Hund" genannt. Auch gilt er als treuer Wächter und ein Sprichwort sagt, der Hund ist ein Hund im Elend seiner Familie. In Deutschland, gilt der Hund auch als treu. 
Eigenschaften der Fabeltiere:

\begin{tabular}{|c|c|c|}
\hline Fabeltiere & Name in der Fabel & Eigenschaften \\
\hline Affe & Martin & intrigant, eitel \\
\hline Bär & $\begin{array}{l}\text { Petz, Meister, } \\
\text { Braun }\end{array}$ & $\begin{array}{l}\text { nett, freundlich, gutmütig, } \\
\text { ein wenig naiv und einfältig }\end{array}$ \\
\hline Biber & Bockert, Bokert & fleißig und arbeitsam \\
\hline Dachs & $\underline{\text { Grimbart }}$ & nachdenklich, ruhig \\
\hline Ente & Tybbke & dumm, einfältig, achtlos \\
\hline Esel & $\begin{array}{l}\text { Langohr, } \\
\text { Boldeqyn }\end{array}$ & störrisch, faul \\
\hline Fuchs & $\begin{array}{l}\text { Reinecke, } \\
\text { Reinhart }\end{array}$ & schlau, listig, durchtrieben \\
\hline $\begin{array}{l}\text { Häher } \\
\text { (Rabenvogel) }\end{array}$ & $\begin{array}{l}\text { Markart, } \\
\text { Markwart }\end{array}$ & vorlaut, aufmüpfig \\
\hline Hahn & Henning & $\begin{array}{l}\text { hochnäsig, eitel, stolz, } \\
\text { hochmütig }\end{array}$ \\
\hline Hase & $\begin{array}{l}\text { Lampe, } \\
\text { Lampe }\end{array}$ & ängstlich, vorsichtig, vorlaut \\
\hline
\end{tabular}




\begin{tabular}{|c|c|c|}
\hline Henne & Kratzefuß & einfach, einfältig, dumm \\
\hline Hund & Hylax & treu, sorglos, freundlich \\
\hline Igel & Arbnora, Swineigel & schlau, klug \\
\hline Kater & $\begin{array}{l}\text { Hinze, Murner, } \\
\text { Murr }\end{array}$ & eigensinnig, sturr \\
\hline Kranich & Lütke & bürokratisch, starrsinnig \\
\hline Krähe & Merkenau & naiv, leichtgläubig \\
\hline$\underset{\text { (Schaf) }}{\text { Lamm }}$ & Lamb & $\begin{array}{l}\text { schutzlos, ohne Rechte, dumm, } \\
\text { fromm, schwach }\end{array}$ \\
\hline Löwe & $\begin{array}{l}\text { Leo, Leu, König } \\
\text { der Tiere, Nobel }\end{array}$ & stolz, königlich, stark, mächtig \\
\hline Luchs & Lynx & vorsichtig, klug \\
\hline Rabe & Pflückebeutel & $\begin{array}{l}\text { besserwisserisch, diebisch, } \\
\text { dumm, eitel }\end{array}$ \\
\hline Storch & $\underline{\text { Adebar, }} \underline{\text { Meister }}$ & $\begin{array}{l}\text { hochmütig, gelehrt, bringt die } \\
\text { Kinder }\end{array}$ \\
\hline Wolf & Isegrim & lügt, gierig, rücksichtslos, böse \\
\hline
\end{tabular}




\begin{tabular}{|l|l|l|}
\hline \hline Ziege & Metke & leichtgläubig, naiv, unzufrieden \\
\hline
\end{tabular}

Es gibt arabische und deutsche Sprichwörter, die den gleichen Sinn haben. Es gibt Sprichwörter, die anderen Sinn, aber ähnliche Eigenschaften von Tieren haben.

Daher werde ich die Symbolik einiger Tiere in arabischer Sprache und die Übersetzung in deutscher Sprache zeigen, zu demonstrieren, dass die Tiersymbolik in Sprichwörtern bei einigen Tieren sowohl in deutscher als auch in arabischer Sprache übereinstimmen kann aber die Tiersymbolik in beiden Sprachen durchaus auch unterschiedlich sein kann. Ich glaube, dass dies auf das sprachliche Erbe, die Kultur, die Legenden oder die Volkserzählung zurückgeführt werden kann. Dies müsste jedoch durch eine andere Arbeit geklärt werden.

Der Stier im arabischen ezeichnet Kraft, Leichtsinn und irrationale Macht und trifft diejenigen, die diese Eigenschaften besitzen (wie einen Stier), d. h. einen starken, der zerstört und schädigt. Er ist gedankenlos und (tobender Stier), d. h. der starke und rücksichtslose Mann.

Der Elefant ist ein Symbol für Taktlosigkeit und tölpelhaft z.B.: Er hat sich wieder mal benommen wie ein Elefant im Porzellanladen. 


\section{Symbolik einiger Tiere in arabischer Sprache und ihre} Übersetzung in deutscher Sprache

\subsection{Symbolik der Hunde in arabischer Sprache und ihre deutsche Übersetzung}

Trotz der Treue und Ehrlichkeit der Hunde versetzen arabische Sprichwörter sie immer in die Position des Abfalls und der Gemeinheit:

\begin{tabular}{|c|c|}
\hline \multicolumn{2}{|l|}{ Arabische Sprichwörter über Hunde } \\
\hline ذيل الكلب عمره ما يتعدل & $\begin{array}{l}\text { Der Schwanz des Hundes wird sich } \\
\text { niemals gerade ausrichten }\end{array}$ \\
\hline الكلاب تعوي والقافلة تسير & $\begin{array}{l}\text { - Die Hunde heulen und der Konvoi } \\
\text { läuft. }\end{array}$ \\
\hline مثل الكلب يعض الأيد إللي اتمدت له & - Beiß nie die Hand, die dich füttert. \\
\hline لو لك حاجة عند الكلب قوله ياسيدي & $\begin{array}{l}\text { Wenn du eine Bitte an einen Hund } \\
\text { hast, sage inm: oh mein Herr. }\end{array}$ \\
\hline 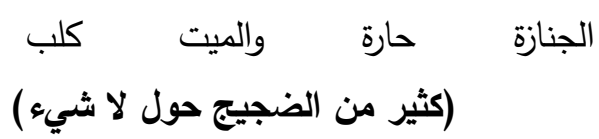 & Viel Lärm um nichts. \\
\hline
\end{tabular}

- Trotz der ihnen zugeschriebenen Unehrlichkeit, sind Wölfe ein Symbol für Stärke und Intelligenz.

Sei ein Wolf, damit dich keine Wölfe fressen تذأب حتى لا تأكلك الذئاب 


\subsection{Symbolik der Affen in arabischer Sprache und ihre deutsche Übersetzung}

Der Affe wurde mit Hässlichkeit und dem Ausdruck hasserfüllter Form in Verbindung gebracht, daher verwendeten die Alten ihn in den folgenden Sprichwörtern:

\begin{tabular}{|c|c|}
\hline \multicolumn{2}{|l|}{ Arabische Sprichwörter über Affen } \\
\hline $\begin{array}{l}\text { Wer den Affen für sein Geld nimmt, } \\
\text { gibt das Geld weg und (doch) bleibt } \\
\text { der Affe derselbe }\end{array}$ & على واخد القرد على ماله يروح المال ويبقى القرد \\
\hline $\begin{array}{l}\text { Der Affe ist wie eine Gazelle in den } \\
\text { Augen seiner Mutter. } \\
\text { Der Affe findet seine eigene junge } \\
\text { Schönste. }\end{array}$ & القرد في عين أمه غزال \\
\hline $\begin{array}{l}\text { Sie werden dich verwechseln, Affe. } \\
\text { Er hat nichts zu verlieren }\end{array}$ & هيسخوك يا قرد غزال \\
\hline Ein Affe unter dem Turban & تحت العمّة قرد \\
\hline
\end{tabular}

\subsection{Symbolik der Katzen in arabischer Sprache und ihre deutsche Übersetzung}

Was Katzen betrifft, so bringt das Erbe sie immer in eine Position des Verrats, der Untreue und der mangelnden Anerkennung der Dankbarkeit. Daher betonen alle Sprichwörter diese Bedeutung. 


\begin{tabular}{|c|c|}
\hline \multicolumn{2}{|l|}{ Arabische Sprichwörter über Katzen } \\
\hline Die Katze liebt es zu würgen & - القط بيحب خناقه \\
\hline Eine Katze hat neun Leben & - قط بسبع أرواح \\
\hline $\begin{array}{l}\text { Wenn die Katze aus dem Haus ist, } \\
\text { tanzen die Mäuse auf dem Tisch. }\end{array}$ & - إن غاب القط العب يا فأر \\
\hline Wie Katzen fressen und leugnen & زي القطط تأكل وتنكر \\
\hline $\begin{array}{l}\text { Wenn man vom Teufel spricht, } \\
\text { kommt er. }{ }^{11}\end{array}$ & جبنا سيرة القط جه ينط \\
\hline Die Katze unter die Tauben setzen. & سلم القط مفتاح الكرار \\
\hline
\end{tabular}

\subsection{Symbolik der Esel in arabischer Sprache und ihre deutsche Übersetzung}

Der Esel hat keinen Willen, immer der Fall in der Hand seines Besitzers

\begin{tabular}{|l|l|}
\hline \multicolumn{2}{|l|}{ Arabische Sprichwörter über Esel } \\
\hline $\begin{array}{l}\text { Einem guten Esel fehlt nie ein } \\
\text { Sattel. }\end{array}$ \\
\hline $\begin{array}{l}\text { Befestigen Sie den Esel da, wo sein } \\
\text { Besitzer will. }\end{array}$ \\
\hline
\end{tabular}

${ }^{11}$ Vgl. Redewendungen: Wörterbuch der deutschen Idiomatik, 2013, S. 749. 
Wenn man dem Teufel den kleinen Finger gibt, dann nimmt der Teufel die ganze Hand.
سكتنا له دخل بحماره:أي سكتنا على دخوله

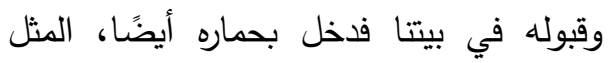

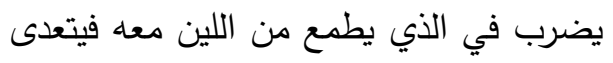
حدوده

Menschen und Tiere haben ähnliche Eigenschaften. Diese Eigenschaften kann man in den verschiedenen Redewendungen bemerken z.B.:

\begin{tabular}{|c|c|}
\hline Deutsch & Arabisch \\
\hline sanft wie ein Lamm & حمل وديع \\
\hline $\begin{array}{l}\text { dastehen wie die Kuh vorm neuen } \\
\text { Tor }\end{array}$ & كأنّ على رؤوسهم الطير اتسمر مكانه \\
\hline $\begin{array}{l}\text { rot wie ein Krebs } \\
\text { (Nach drei Stunden in der Sonne war } \\
\text { er rot wie ein Krebs.) }\end{array}$ & $\begin{array}{l}\text { وشه احمر (تعبير (Ausdruck von } \\
\text { Sein Gesicht ist rot (تخل) } \\
\text { Schüchternheit) }\end{array}$ \\
\hline stark wie ein Pferd & (رجل) 24حصان \\
\hline schwimmt wie ein Fisch & بتعوم مثل السمكة \\
\hline schwarz wie ein Rabe & الغراب رمزالتشاؤم \\
\hline störrisch wie ein Esel & عنيد وغبي مثل الحمار \\
\hline schlau wie ein Fuchs & شخص تعلب أومكار \\
\hline
\end{tabular}




\begin{tabular}{|c|c|}
\hline $\begin{array}{l}\text { Jedem Tierchen sein Pläsierchen! } \\
\text { »Jeder nach seinem } \\
\text { Geschmack«, »Die Geschmäcker } \\
\text { sind verschieden« und »Über } \\
\text { Geschmack lässt sich streiten«. }\end{array}$ & 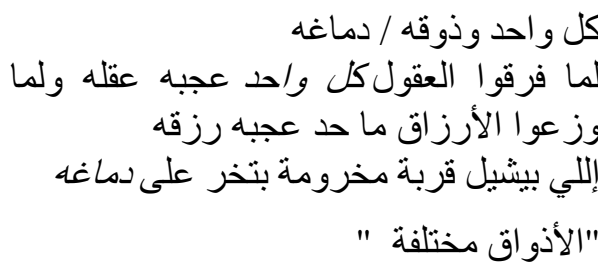 \\
\hline $\begin{array}{l}\text { schlafen wie ein Murmeltier } \\
\text { (sehr tief und fest schlafen) }\end{array}$ & نام مثل الخروف(غرق في النوم) \\
\hline sich zum Affen machen & زي فرقعلوز \\
\hline Ein Fisch auf dem Trockenen & المناسب لل (يشعر مالة شخص سمكة ليس في اليابسة) \\
\hline
\end{tabular}

Einige Eigenschaften z.B.: Dummheit, Schlauheit der Feigheit werden bei einigen Tieren bei den Arabern anders als bei den Deutschen belegt.

Tierische Sprichwörter sind eine der verschiedenen Gruppen des Phraseologiebestandes im Deutschen und Arabischen. Sie spiegeln die Überwachung der Leute über die Erscheinung und das Verhalten der Tiere wider und sind von daher der kulturell belehrend Träger jeder Sprache.

Ich denke, dass meine Hypothese richtig ist, dass es gleiche arabische und deutsche tierische Sprichwörter gibt. Einige Sprichwörter haben schon ähnliche Bedeutung, aber verschiedene Eigenschaften von Tieren. Es gibt auch nur 
arabische tierische Sprichwörter und nur deutsche tierische Sprichwörter.

\section{Schlussfolgerung}

Als Ergebnis der Analyse, des Vergleichs und der Gegenüberstellung deutscher und arabischer Sprichwörter stellten wir fest, dass die Bilder von Haustieren unterschiedliche emotionale Belastungen tragen. In Anbetracht der Sprichwörter und Sprüche zweier Sprachen haben wir ein positives Bild davon, Sprichwörter über Tiere zu vergleichen. Deutsche und Araber haben ihre eigene Herangehensweise an Sprichwörter über Tiere. In arabischen Sprichwörtern und Sprüchen haben wir die Übertragung von Bildern von Haustieren auf Menschen festgestellt. In deutschen Sprichwörtern und Sprüchen haben wir die Gebrauchstauglichkeit deutscher Sprichwörter für Araber festgestellt, da sie einander ähnlich sind. Daraus können wir schließen: Deutsche Sprichwörter können ähnlich sein wie arabische nur in einem anderen Sinne, und die Bedeutung der Sprichwörter ist dieselbe!

Einige Eigenschaften z.B.: Dummheit, Schlauheit, oder Feigheit werden jedoch bei einigen Tieren bei den Arabern anders als bei den Deutschen gebraucht. Vielleicht hängt das von der Kultur, den Legenden und der Volkserzählung ab. 


\section{Literaturverzeichnis}

- Andre Jolles, Einfache Formen, Tübingen: Niemeyer 1930 (Neudruck, 1965), S. $158 \mathrm{f}$.

- Burger, Harald: Idiomatik des Deutschen. Tübingen: Niemeyer, 1978.

-Burger, Harald: Phraseologie: eine Einführung am Beispiel des Deutschen. Berlin: Schmidt, 1998.

- Dietmar Peil: Sprichwort. In: Volker Meid (Hrsg.), Sachlexikon Literatur, München 2000, S. 855 - 857; S. 855 , Spalte 1

- Duden; Bd. 11 (Redewendungen. Wörterbuch der deutschen Idiomatik. Mannheim: Brockhaus, 2002.

- Duden. Redewendungen und sprichwörtliche Redensarten. Band II.Mannheim-Leipzig- Zürich; Duden, 1997

- Elisabeth Vollers-Sauer: Sprichwort. In: Helmut Glück (Hrsg.), Metzler Lexikon Sprache, stuttgart, Weimar 2000, S. 685 - 686; S. 685, Sp. 2

- Erhard Agricola u. a., Die deutsche Sprache, Leipzig: Bibliographi, sches Institut 1969, Bd. 1, S. 594.Friedrich Seiler, Deutsche Sprichwörterkunde, München: Beck 1922, (Neudruck 1967), S. 2, S. 11 f, S. 592 f.

- Fleischer, Wolfgang: Phraseologie der deutschen Gegenwartssprache. Leipzig: VEB Bibliographisches Institut, 1982.

- Földes, Csaba: Sind alle deutschen Redensarten wirklich deutsch? In: Sprachpflege (1984/ 9). S. 127 - 129. 
- Görner, Herbert: Kleine Idiomatik der deutschen Sprache. Leipzig: VEB Bibliographisches Institut, 1979

- Harald Burger, Idiomatik des Deutschen, Tübingen: Niemeyer 1973, S. 54.

- Hasubek, Peter: Einleitung. In: Fabelforschung. Hg. v. Peter Hasubek. Darmstadt: Wissenschaftliche Buchgesellschaft, 1983 (Wege der Forschung; 572). S. 4.

- Hermann Bausinger, Formen der »Volkspoesie«, Berlin: E. Schmidt, 1968 , S. 98 , S. $93-95$.

- Hain, Mathilde: Das Sprichwort. In: Ergebnisse der Sprichwörterforschung. Hrsg. v. Wolfgang Mieder. Bern: Lang, 1978. S. $13-25$.

- Honcamp, Franz C.: Das Sprichwort, sein Werth und seine Bedeutung. In: Deutsche Sprichwörterforschung des 19. Jahrhunderts. Hrsg. v. Wolfgang Mieder. Bern: Lang, 1984. S. 45 - 66.

- Koller, Werner: Redensarten: linguist. Aspekte, Vorkommensanalysen, Sprachspiel. Tübingen: Niemeyer, 1977.

- Küpper, H. Wörterbuch der deutschen Umgangssprache / H. Küpper Claassen Verlag, Hamburg, 1963. - 163 S.

- Lutz Röhrich, Lexikon der sprichwörtlichen Redensarten, Freiburg, i. Br.: Herder 1973, Bd. 1, S. 9.

- Lutz Röhrich u. Wolfgang Mieder, Sprichwort, Stuttgart: Metzler 1977, S.3.

- Mathilde Hain, Sprichwort und Volkssprache, Gießen:Schmitz, 1951, S.36-38.

- Matti Kuusi, Parömiologische Betrachtungen, Helsinki: Academia, Scientiarum Fennica 1957, S. 52. 
- Mieder, Wolfgang: Sprichwörter im modernen Sprachgebrauch. In: Deutsche Sprichwörter in Literatur, Politik, Presse und Werbung. Hamburg: Buske, 1983. S. 53 - 76.

- Mieder, Wolfgang: Karl Kraus und der sprichwörtliche Aphorismus. In: Deutsche Sprichwörter in Literatur, Politik, Presse und Werbung. Hamburg: Buske, 1983. S. 113 - 129.

- Mieder, Wolfgang: Rund um das Sprichwort „Morgenstunde hat Gold im Munde“. In: Deutsche Sprichwörter in Literatur, Politik, Presse und Werbung. Hamburg: Buske, 1983. S. $105-113$.

- Neue Scheidemünze. Aus dem Deutschen Sprichwörter - Lexikon des Karl Friedrich Wilhelm Wander. Berlin: Volk und Wissen, 1987.

- Palm, Christine: Phraseologie: eine Einführung. Tübingen: Narr, 1995.

- Redewendungen: Wörterbuch der deutschen Idiomatik, 2013, S. 749

- Robert Petsch, Spruchdichtung des Volkes, Halle: Niemeyer 1938, S. 108.

- Röhrich, Lutz: Sprichwoertliche Redensarten aus Volkserzaehlungen. In: Ergebnisse der Sprichwörterforschung. Hrsg. v. Wolfgang Mieder. Bern: Lang, 1978. S. $121-141$.

- Simrock, Karl: Die deutschen Sprichwörter. Einleitung v. Wolfgang Mieder. Stuttgart: Reclam, 1988.

- Spieß, Karl: Das Sprichwort. In: Deutsche Sprichwörterforschung des 19. Jahrhunderts. Hrsg. v. Wolfgang Mieder. Bern: Lang, 1984. S. $261-285$. -Urbas, Wilhelm: Die Sprichwörter und ihre Entstehung. In: Deutsche Sprichwörterforschung des 19. Jahrhunderts. Hrsg. v. Wolfgang Mieder. 
Bern: $\quad$ Lang, $\quad 1984 . \quad$ S. $\quad 81 \quad-108$.

- Wander, Karl Friedrich Wilhelm: Das Sprichwort, betrachtet nach Form und Wesen, für Schule und Leben, als Einleitung zu einem großen volksthümlichen Sprichwörterschatz. Nachdruck der Ausg. v. 1836. Hrsg. und eingeleitet v. Wolfgang Mieder. Bern: Lang, 1983.

- Werner Herzenstiel, Erziehungserfahrung im deutschen Sprichwort, Saarbrücken: Universitäts- und Schulbuchverlag 1973, S. 65.

\section{Internetquellen}

https://www.phraseo.de/sammlung/tiere

http://www.kuriosetierwelt.de/tier-symbolik-bienen-sind-fleissig-undesel-sind-dumm

https://www.phase-6.de/magazin/rubriken/sprichwoerter/warumsagen-wir-eigentlich-redewendungen-rund-um-tiere https://www.br.de/wissen/sprichwoerter-redensarten-redewendungentiere-100.html

https://www.duda.news/wissen/tierische-redewendungen-und-ihrebedeutungen

\section{المراجع العربية}

الأمثال العامية - مشروحة ومرتبة حسب الحرف الأول من المثل - احمد تيمور باشا - الأمثال العامية

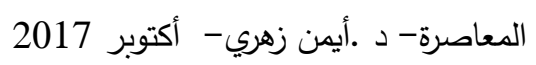

الدثل السائر في أدب الكاتب والثاعر لضياء الدين بن الأثير -تحقيق الدكتور أحمد الحوفي وبدوي طبانة -

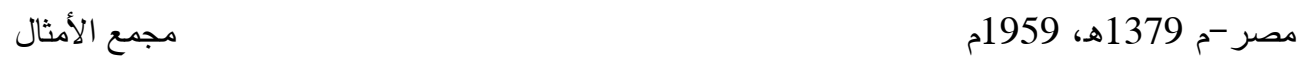

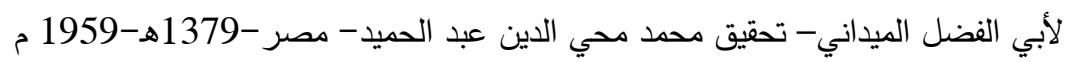

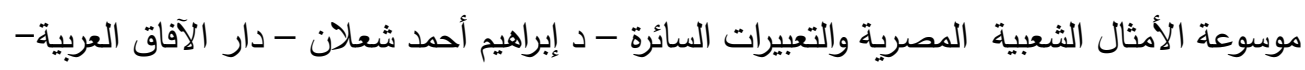
الطبعة الأولى - 1423هـ، 2003م. 